\title{
First-principles study of optical, elastic anisotropic and thermodynamic properties of TiN under high temperature and high pressure
}

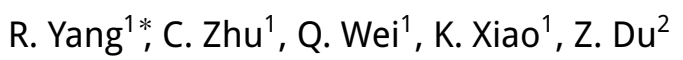 \\ 1 School of Physics and Optoelectronic Engineering, Xidian University, Xi'an, Shaanxi 710071, PR China \\ 2 National Supercomputing Center in Shenzhen, Shenzhen 518055, PR China
}

Received November 24, 2016, in final form April 18, 2017

\begin{abstract}
The optical, elastic anisotropic and thermodynamic properties of TiN in the $\mathrm{NaCl}$ (B1) structure are analyzed in detail in the temperature range from 0 to $2000 \mathrm{~K}$ and the pressure range from 0 to $20 \mathrm{GPa}$. From the calculated dielectric constants, a first order isostructural phase transition between 29 and $30 \mathrm{GPa}$ is found for TiN. The absorption spectra exhibit high values ranging from the far infrared region to the ultra-violet one. The anisotropy value of Young's modulus of TiN is smaller than that of c-BN at $0 \mathrm{GPa}$ and the anisotropy of TiN clearly increases with an increase of pressure. The effects of pressure and temperature on the bulk modulus, Grüneisen parameter, Gibbs free energy, and Debye temperature are significant. The Grüneisen parameter of TiN is much larger than that of c-BN. At temperatures below $1000 \mathrm{~K}$, TiN's heat capacity is much larger than that of c-BN.
\end{abstract}

Key words: TiN, optical properties, elastic anisotropy, thermodynamic properties, first-principles

PACS: $61.82 . B g, 62.20 . d c, 71.20 . B e, 71.15 . M b$

\section{Introduction}

As one of transition-metal nitrides, titanium nitride (TiN) was first separated by Story-Maskelyne from a meteorite. It crystallizes into the well-known rock-salt structure. It is one of the important metal cutting tools and coating materials for surface protection due to its high melting temperature, extreme hardness and fine corrosion resistance [1-6]. Its ceramics have a good resistance to corrosion by liquid steel for some steel making processes [7]. It is also used for diffusion barriers, superconducting devices, and energy-saving coatings for windows due to their strong infrared reflection [8]. The high-pressure and high-temperature studies on various materials are very important both from basic and applied point of view. Up to now, some elastic and thermodynamic properties of $\mathrm{TiN}$ in the $\mathrm{NaCl}$ (B1) structure have been calculated. Some mechanical properties of TiN were studied in [3, 4, 9]. Liu et al. studied the pressure and temperature dependences of the relative volume, the Debye temperature, heat capacity and thermal expansion for TiN [10]. An anomalous volume behavior of TiN between 7.0 and $11.0 \mathrm{GPa}$ was found [11], which was interpreted as a first order isostructural phase transition. Recently, a phase transformation between $35 \mathrm{GPa}$ and $57 \mathrm{GPa}$ was shown by the volume collapse of TiN [12].

The effect of the first order isostructural phase transition on the optical properties of TiN has not been reported so far. Thus, the optical properties are analyzed in our works by first-principles calculations. Moreover, the relationship between Young's modulus anisotropy and pressure is discussed. Several thermodynamic properties of TiN are analyzed in detail at higher temperature and pressure. To the best of our knowledge, these properties have not been discussed in previous literature. As one of the groups III-nitrides semiconductor compounds, cubic boron nitride (c-BN) exhibits many fascinating mechanical

*E-mail: yrk18687@163.com 
and thermal properties, such as, extreme hardness, high melting point, high thermal conductivity, and so on. Thus, some properties of c-BN are also calculated for comparison.

\section{Computational methods}

Structural optimization and prediction of properties are performed using the density functional theory (DFT) with the generalized gradient approximation (GGA) parameterized by Perdew, Burke and Ernzerrof (PBE) as implemented in the Cambridge Serial Total Energy Package (CASTEP) code [13]. The optical properties are analyzed using PBE0 hybrid functional. The cut-off energy determines the numbers of plane waves, while the number of special k-points is used for the Brillouin zone (BZ) division [14]. Convergence tests prove that the Brillouin zone sampling and the kinetic energy cut-off are reliable to guarantee excellent convergence [15]. For the geometry optimization, the self-consistent field tolerance threshold is taken as $5 \times 10^{-7} \mathrm{eV} /$ atom. The convergent value of the total energy difference is less than $5 \times 10^{-6} \mathrm{eV} /$ atom. The maximum Hellmann-Feynman force is given as $0.01 \mathrm{eV} / \mathrm{A}$. The maximum stress is taken as less than $0.02 \mathrm{GPa}$. The maximum atom displacement is smaller than $5 \times 10^{-3} \AA$. The energy cut-off values are $580 \mathrm{eV}$ for TiN and $550 \mathrm{eV}$ for c-BN. The k-points in the first Brillouin zone are $14 \times 14 \times 14$ for TiN and c-BN. The elastic constants are calculated by using the stress-strain methods. In the strain calculation, the number of steps is set as 6. Maximum strain amplitude is taken as 0.003 . In the calculations of the optical properties, the norm-conserving pseudo-potentials have been applied to all the ion-electron interactions. The scissors operator is taken as $0.5 \mathrm{eV}$. The instrumental smearing (which describes the Gaussian broadening to be used for calculating the dielectric function) is set to $0.2 \mathrm{eV}$ and the incident light direction is (100).

The optical properties are analyzed for TiN, based on GGA-PBE and PBE0 calculations. Generally, the optical properties of systems are evaluated by the complex dielectric function, which is dependent on frequency and is as follows:

$$
\varepsilon(\omega)=\varepsilon_{1}(\omega)+\mathrm{i} \varepsilon_{2}(\omega) .
$$

The complex dielectric function is mainly connected to the electronic structures. The imaginary part of the dielectric function, $\varepsilon_{2}(\omega)$, is calculated based on the momentum matrix elements between the occupied and unoccupied wave functions, it is as follows [16]:

$$
\varepsilon_{2}(\omega)=\frac{V e^{2}}{2 \pi \hbar m^{2} \omega^{2}} \int \mathrm{d}^{3} k \sum_{n, n^{\prime}}\left|\left\langle k n|p| k n^{\prime}\right\rangle\right|^{2} f(k n)\left[1-f\left(k n^{\prime}\right)\right] \delta\left(E_{k n}-E_{k n^{\prime}}-\hbar \omega\right),
$$

where $V$ is the unit cell volume, $e$ is electronic charge, $n$ and $n^{\prime}$ are the initial state and final state band indexes, respectively, $p$ denotes the momentum operator, $|k n\rangle$ expresses a crystal wave function, $f(k n)$ represents the Fermi distribution function, and the energy of the incident photon is $\hbar \omega$. The real part, $\varepsilon_{1}(\omega)$, is given by Kramers-Kroning relationship:

$$
\varepsilon_{1}(\omega)=1+\frac{2}{\pi} M \int_{0}^{\infty} \frac{\varepsilon_{2}\left(\omega^{\prime}\right) \omega^{\prime}}{\omega^{\prime 2}-\omega^{2}} \mathrm{~d} \omega^{\prime},
$$

where $M$ is the principal value of the integral. The real part of dielectric function is in relation to the electric polarization characteristics of the material. Optical reflectivity, $R(\omega)$, and absorption coefficient, $I(\omega)$, can be calculated based on the complex dielectric function $\varepsilon(\omega)$, respectively. These expressions are shown as follows [17, 18]:

$$
\begin{aligned}
R(\omega) & =\frac{(n-1)^{2}+k^{2}}{(n+1)^{2}+k^{2}}, \\
I(\omega) & =\frac{2 \omega k(\omega)}{c} .
\end{aligned}
$$

An illustrative way of describing the elastic anisotropy is a three-dimensional surface representation. It shows the variation of the elastic modulus with crystallographic direction. This directional dependence 
of the Young's modulus $E$ for a cubic crystal is given by [19]:

$$
\frac{1}{E_{\text {Cubic }}}=s_{11}-2\left(s_{11}-s_{12}-\frac{1}{2} s_{44}\right)\left(l_{1}^{2} l_{2}^{2}+l_{2}^{2} l_{3}^{2}+l_{3}^{2} l_{1}^{2}\right) \text {. }
$$

Based on the optimized crystal structures, the thermodynamic properties for TiN under high temperature and high pressure are calculated by means of the quasi-harmonic Debye model, in which the phonon effect is considered and the results are achieved by GIBBS program [20]. The temperature is included in the quasi-harmonic Debye approximation. In fact, thermodynamic properties of the crystal can be obtained by treating the lattice vibrations as a quantized phonon. The model has been successfully used to predict the thermodynamic properties of some materials [21-23]. In the quasi-harmonic Debye model, the non-equilibrium Gibbs function $G^{*}(V ; P, T)$ takes the form of [24]

$$
G^{*}(V ; P, T)=E(V)+P V+A_{\mathrm{Vib}}\left(\Theta_{\mathrm{D}}(V) ; T\right),
$$

where $E(V)$ is the total energy per unit cell, $P V$ corresponds to the constant hydrostatic pressure condition, $\Theta_{\mathrm{D}}(V)$ is the Debye temperature, $T$ is the absolute temperature and $A_{\mathrm{Vib}}$ is the vibrational contribution, that can be written as follows:

$$
A_{\mathrm{Vib}}\left(\Theta_{\mathrm{D}} ; T\right)=n k T\left[\frac{9}{8} \frac{\Theta_{\mathrm{D}}}{T}+3 \ln \left(1-\mathrm{e}^{-\Theta_{\mathrm{D}} / T}\right)-D\left(\Theta_{\mathrm{D}} / T\right)\right],
$$

where $n$ is the number of atoms in a chemical formula, $D\left(\Theta_{\mathrm{D}} / T\right)$ represents the Debye integral with Poisson ratio $\sigma$. The $\Theta_{\mathrm{D}}$ is expressed by

$$
\Theta_{\mathrm{D}}=\frac{\hbar}{k}\left(6 \pi^{2} V^{1 / 2} n\right)^{1 / 3} f(\sigma) \sqrt{\frac{B_{S}}{M}},
$$

where $M$ is the molecular mass per unit cell, $B_{S}$ is the adiabatic bulk modulus, which is equal to the isothermal bulk modulus $B_{T}$ in the Debye model, leading to the following equation:

$$
B_{S}=B_{T}=V \frac{\mathrm{d}^{2} E(V)}{\mathrm{d} V^{2}},
$$

where $E$ is the total energy of the crystal at $0 \mathrm{~K}$.

For the non-equilibrium Gibbs function, $G^{*}(V ; P, T)$, can be minimized with respect to volume $V$. There is

$$
\left[\frac{\partial G^{*}(V ; P, T)}{\partial V}\right]_{P, T}=0 .
$$

By solving the equation 2.11 with respect to $V$, a thermal equation of state can be gotten. The heat capacity, $C_{V}$, and thermal expansion coefficient, $\alpha$, are given as follows:

$$
\begin{aligned}
C_{V} & =3 n k\left[4 D(\Theta / T)-\frac{3 \Theta / T}{\mathrm{e}^{\Theta / T}-1}\right], \\
\alpha & =\frac{\gamma C_{V}}{B_{T} V} .
\end{aligned}
$$

Anharmonic effect of the vibrating lattice is usually described in terms of Grüneisen parameter, $\gamma$, which can be defined as

The heat capacity $C_{P}$ is expressed as

$$
\gamma=-\frac{\mathrm{d} \ln \Theta(V)}{\mathrm{d} \ln V} .
$$

$$
C_{P}=C_{V}(1+\alpha \gamma T) .
$$

The entropy, whose change between states is defined as the integral of the ratio of the reversible heat transfer to the absolute temperature, is a measure of the state of disorder of the system. Entropy $\left(S_{\mathrm{Vib}}\right)$ in the quasi-harmonic Debye model is given by [20]

$$
S_{\mathrm{Vib}}=n k\left[4 D(\Theta / T)-3 \ln \left(1-\mathrm{e}^{-\Theta / T}\right)\right] .
$$




\section{Results and discussion}

\subsection{Optical properties}

Dielectric function, being the bridge between the microscopic physical process and the electronic structure of solid by electronic inter-band transition, reflects the energy band structure of solid materials and other optical information. Figure 1 presents the real part of the calculated dielectric function from 0 to $40 \mathrm{GPa}$ for TiN. The calculated static dielectric constants $\varepsilon(0)$ are 20.4, 19.8, 19.4, 41.2 and 39.4 when the pressures are $0,20,29,30$ and $40 \mathrm{GPa}$, respectively. The $\varepsilon(0)$ decreases with an increase of pressure from 0 to $29 \mathrm{GPa}$, but it shows an anomalous behavior between 29 and $30 \mathrm{GPa}$, which is interpreted as a first order isostructural phase transition. The real part of dielectric function enhances with increasing photon energy and gets to the highest values at about $0.6 \mathrm{eV}$, and afterwards it is a damped oscillation. In the following work, we only study the properties of TiN from 0 to $20 \mathrm{GPa}$.

The calculated results in figure 2 (a) and (b) reveal that TiN possesses a broad frequency response. It has strong reflectivity and absorption both in the low-frequency and high-frequency region. The GGA results are plotted and compared with the PBE0 results. The reflectivity spectra of TiN are characterized by six main peaks. The GGA results show that the peaks are located at 1.0, 11.2, 20.0, 23.3, 37.5 and $40.9 \mathrm{eV}$ at zero pressure, while the PBE0 results show they are located at 1.0, 14.1, 23.3, 25.8, 42.2 and $45.4 \mathrm{eV}$.

The optical absorption spectra are directly connected to the imaginary part of the refractive index,

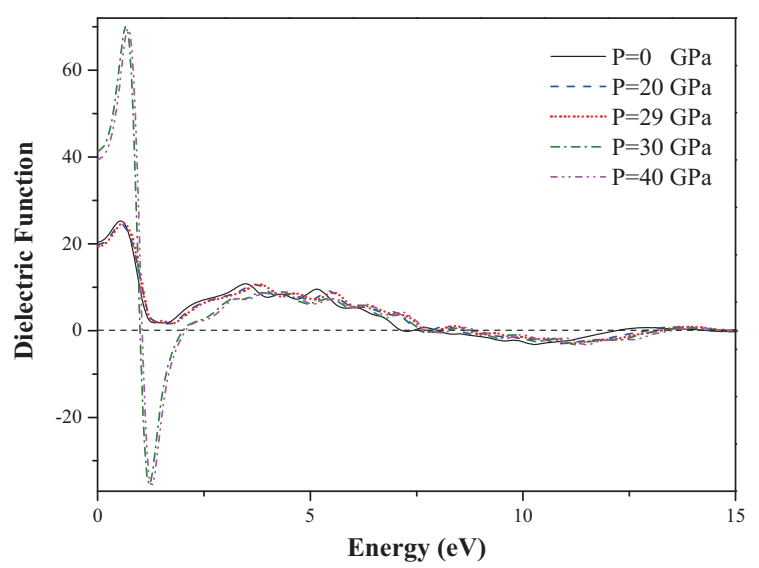

Figure 1. (Color online) Real part of the dielectric function calculated by GGA for TiN.
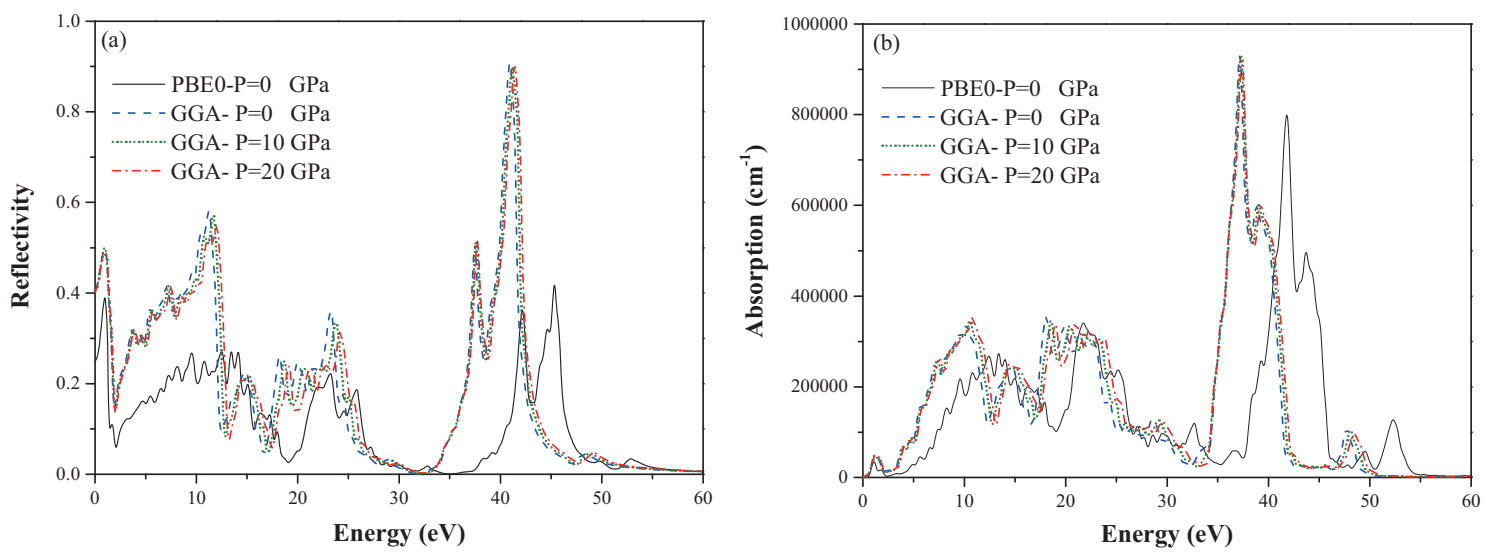

Figure 2. (Color online) (a) Reflectivity $R(\omega)$ and (b) absorption coefficient $I(\omega)$ by GGA-PBE and PBE0 from 0 to $20 \mathrm{GPa}$ for TiN. 
which represents the fraction of the energy lost when a light wave passes through materials. When materials absorb energy, their electrons jump up to higher energy levels. Therefore, the interband transitions in different energy regions are responsible for the pronounced peaks of absorption spectra. The absorption spectra exhibit high values ranging from the far infrared region to the ultra-violet one. One can notice that the absorption peaks are positioned at 10.20, 14.6, 21.3 and $38.9 \mathrm{eV}$ according to GGA, and 15.0, 21.8 and $41.8 \mathrm{eV}$ according to PBE0, in figure 2 (b).

\subsection{Elastic properties}

The elastic properties of a solid are of importance. They are not only closely related to various fundamental solid-state phenomena, such as atomic bonding, equations of state and phonon spectra but are also thermodynamically related to specific heat, thermal expansion, Debye temperature, Grüneisen parameter, and so on [25]. Moreover, knowledge of the elastic constants is very essential for many practical applications related to the mechanical properties of a solid: such as load deflection, thermo-elastic stress, internal strain, sound velocities, and fracture toughness.

In order to study the mechanical stability of TiN, we calculated the second-order elastic constants $C_{i j}$ from 0 to $20 \mathrm{GPa}$ by using the "stress-strain method", which are listed in table 1 . The results of c-BN and TiN at $0 \mathrm{GPa}$ are close to the available data [19,26]. There are three independent elastic constants $C_{11}, C_{12}$ and $C_{44}$. The elastic constant $C_{11}$ represents the resistances to linear compression at $x, y$ and $z$ directions, the elastic constants $C_{12}$ and $C_{44}$ are related to the elasticity in shape. For TiN, $C_{11}$ is the highest in all the elastic constants, which implies that $\mathrm{TiN}$ is incompressible along the coordinate axis.

Table 1. The elastic constants $C_{i j}$ of c-BN and TiN.

\begin{tabular}{|l||l|l|l|l|}
\hline \hline & $\mathrm{P}(\mathrm{GPa})$ & $C_{11}$ & $C_{12}$ & $C_{44}$ \\
\hline \hline \multirow{3}{*}{$\mathrm{c}-\mathrm{BN}$} & 0 & 779 & 165 & 446 \\
\cline { 2 - 5 } & $0[27]$ & 779 & 165 & 446 \\
\cline { 2 - 5 } & $0[26]$ & 798 & 172 & 469 \\
\hline \multirow{4}{*}{ TiN } & 0 & 581 & 126 & 166 \\
\cline { 2 - 5 } & $0[19]$ & 590 & 145 & 169 \\
\cline { 2 - 5 } & 10 & 676 & 141 & 172 \\
\cline { 2 - 5 } & 20 & 767 & 155 & 176 \\
\hline \hline
\end{tabular}

The directional dependence of the Young's modulus of c-BN and TiN is illustrated in figure 3 (a)-(d). The 3D figures of the Young's moduli show the deviation in shape from the sphere and the results indicate that the Young's moduli are anisotropic. In addition, the Young's moduli maximal values of c-BN and $\mathrm{TiN}$ at $0 \mathrm{GPa}$ are 955 and $536 \mathrm{GPa}$, while the minimal values are 721 and $415 \mathrm{GPa}$. Thus, the ratios of the maximal and minimal values are 1.32 and 1.29. So, the anisotropy value of Young's modulus of TiN is smaller than that of c-BN. At 10 and $20 \mathrm{GPa}$ pressure, the Young's moduli maximal values of TiN are 629 and 715, respectively, while the minimal values are 437 and 454 . The anisotropy values are 1.44 and 1.58. Thus, the Young's moduli anisotropy of TiN increases clearly with an increase of pressure.

\subsection{Thermodynamic properties}

Thermodynamic properties of $\mathrm{TiN}$ are determined in the temperature range from 0 to $2000 \mathrm{~K}$, meanwhile, the pressure effects are also analyzed in the range from 0 to $20 \mathrm{GPa}$. By using the quasiharmonic Debye model, thermal expansion coefficients and volumes of TiN at a given pressure and temperature conditions are calculated and the results are consistent with [10].

Heat capacity is an important parameter in condensed matter physics. It provides an essential information on the heat transition process and vibration properties. The relationships between isobaric heat capacity $C_{P}$ and constant volume heat capacity $C_{V}$ versus temperature are plotted in figure 4 Our calculated $C_{V}$ [in figure 4 (b)] of $\mathrm{TiN}$ at $0 \mathrm{GPa}$ is consistent with the results in [10]. The difference 

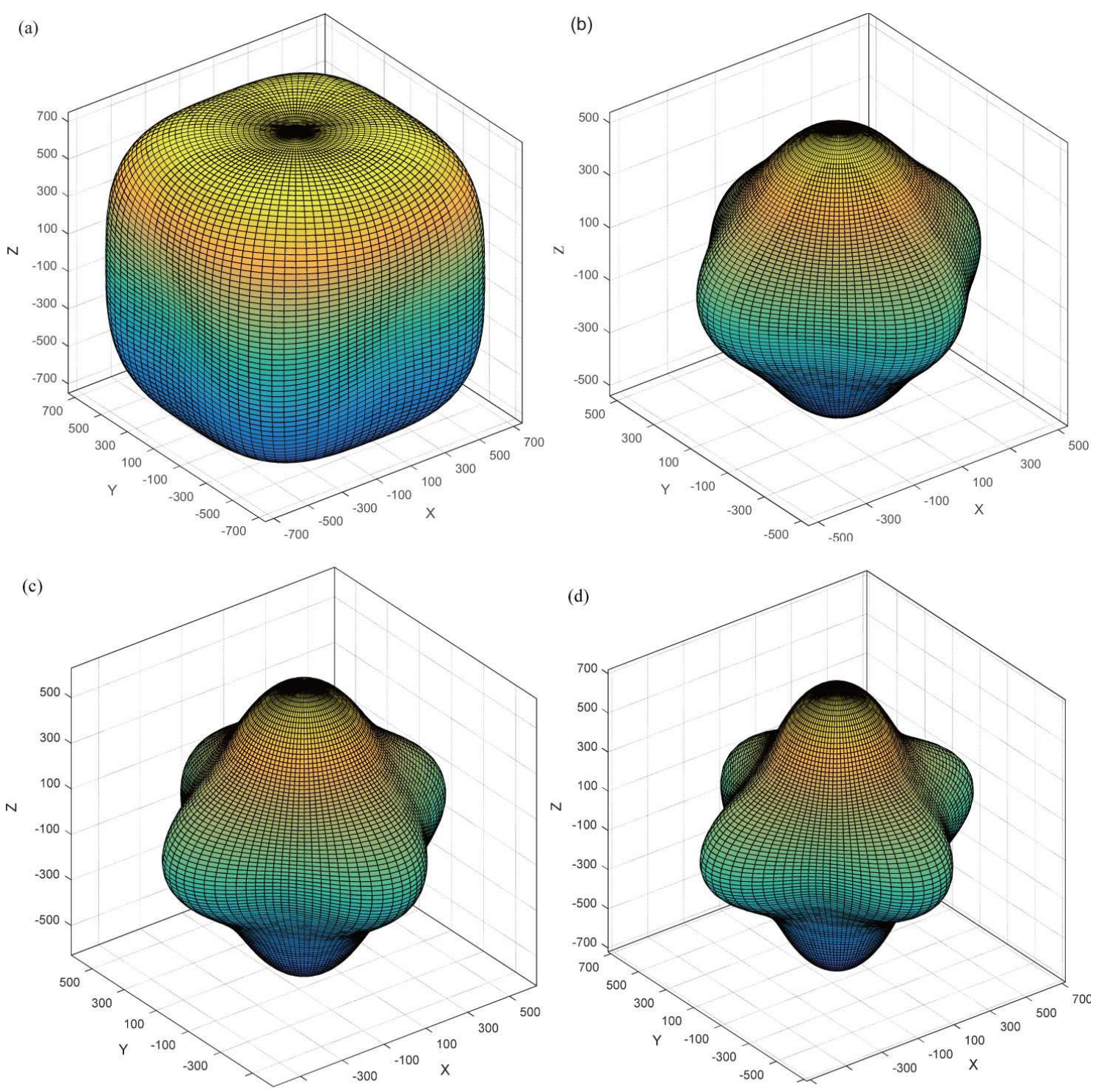

Figure 3. (Color online) The anisotropy of the Young's moduli for (a) c-BN at $0 \mathrm{GPa}$, (b) TiN at $0 \mathrm{GPa}$, (c) TiN at $10 \mathrm{GPa}$ and (d) TiN at $20 \mathrm{GPa}$.

between $C_{P}$ and $C_{V}$ is very small, nearly below $500 \mathrm{~K}$ for c-BN and $400 \mathrm{~K}$ for TiN. Both $C_{P}$ and $C_{V}$ increase rapidly with temperature due to the anharmonic effect. At high temperature ranges, $C_{V}$ approaches a constant value, which is called a Dulong-Petit limit. The $C_{P}$ still increases monotonously with increments of temperature. Both $C_{P}$ and $C_{V}$ increase with the temperature rise at a given pressure and slightly decrease with the pressure rise at a given temperature. The effects of temperature on the heat capacity are very significant. In addition, at lower temperatures, the effects of temperature on TiN's heat capacity are stronger than those of the temperature on c-BN's. At temperature below $1000 \mathrm{~K}$, TiN's heat capacity is larger than that of c-BN. Also, the Dulong-Petit limit of TiN is a little larger than that of c-BN.

The Grüneisen parameter $\gamma$ describes the variation in vibration of a crystal lattice based on the changes of volume or temperature. Recently, it has been widely used to characterize and extrapolate the thermodynamic properties of materials at high pressures and high temperatures, such as the temperature dependence of phonon frequencies and line-widths. It is dominated by lower-frequency transverse modes at low temperatures. The variation of Grüneisen parameter with pressure and temperature is displayed 

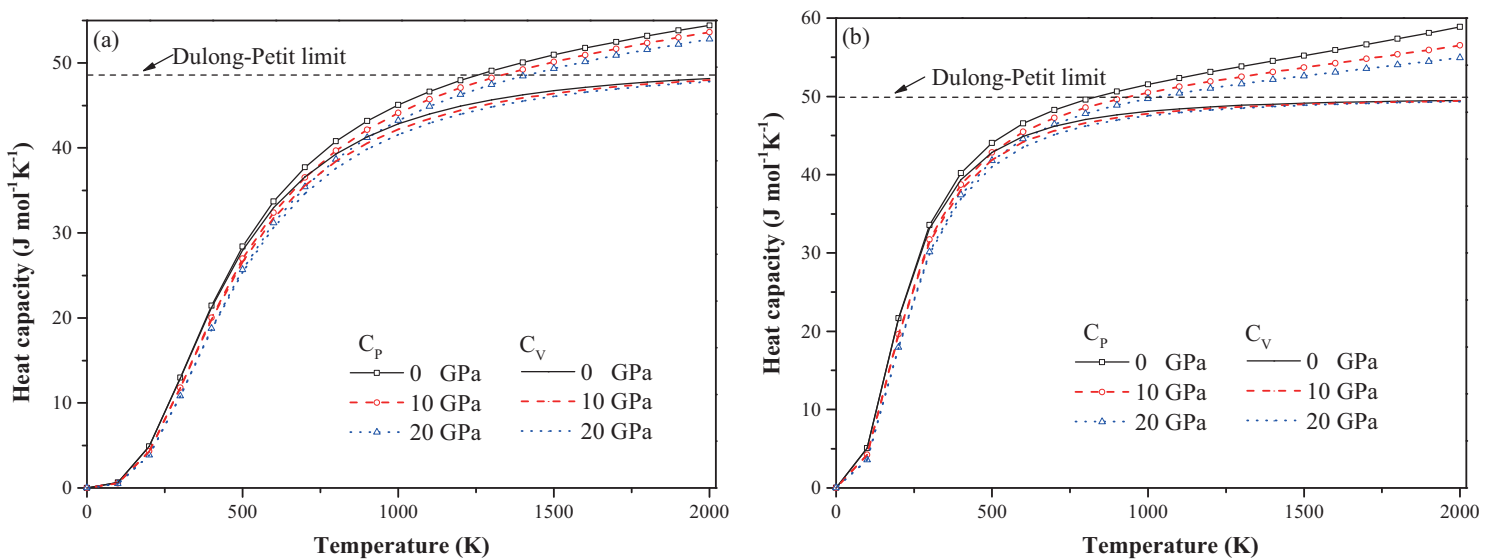

Figure 4. (Color online) Temperature dependence of the heat capacity at different pressures for (a) c-BN and (b) TiN.
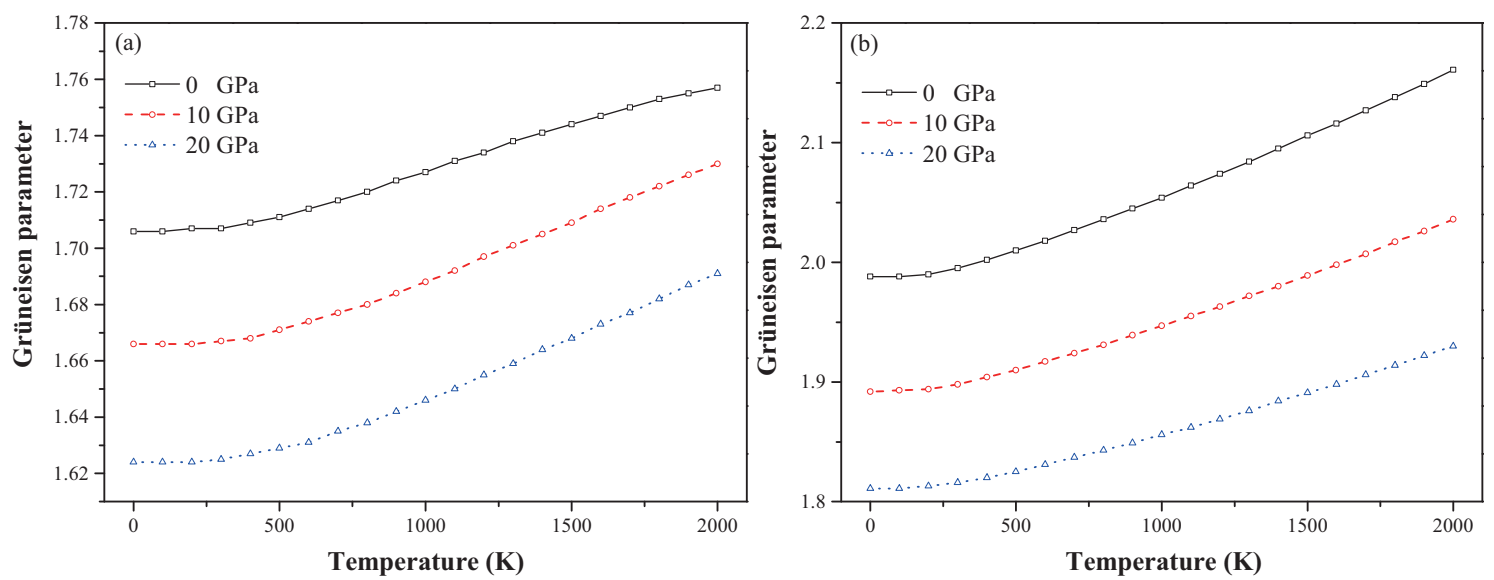

Figure 5. (Color online) The Grüneisen parameter versus temperature of (a) c-BN and (b)TiN.

in figure 5 The $\gamma$ values rapidly decrease with an increase of pressure and increase as temperature increment. The effect of pressure and temperature on TiN's Grüneisen parameter is more significant than that of c-BN. The Grüneisen parameter of TiN is larger than that of c-BN.

The entropy of a system is a measure of the amount of molecular disorder within the system. A system can only generate, rather than destroy, entropy. The entropy of a system can be increased or decreased by energy transports across the system boundary [28]. For TiN, the results of entropy as a function of temperature are shown in figure 6. It can be unambiguously seen that entropy increases monotonously with an increasing temperature at a given pressure and decreases with pressure rise at a given temperature. The effect of temperature on entropy is obvious.

The bulk modulus, $B$, can describe the elasticity of homogeneous isotropic solids, which can be expressed as the force per unit area, and it indicates the compressibility. The relationship between the bulk modulus and temperature at different pressures is shown in figure 7 . These results indicate that $B$ decreases with temperature at a given pressure and rapidly increases with pressure at a given temperature. The effects of pressure and temperature on the bulk modulus are obvious.

Free energy is the inner energy in a thermodynamic system which can be transformed into external work at a thermodynamic process. Gibbs free energy is a kind of free energy. Figure 8 presents the relationships between the Gibbs free energy and temperature at pressure range of 0-20 GPa. The Gibbs free energy is determined by enthalpy, entropy and temperature. The results show that Gibbs free energy decreases with temperature rise. The effect of pressure on the Gibbs free energy is also significant. 


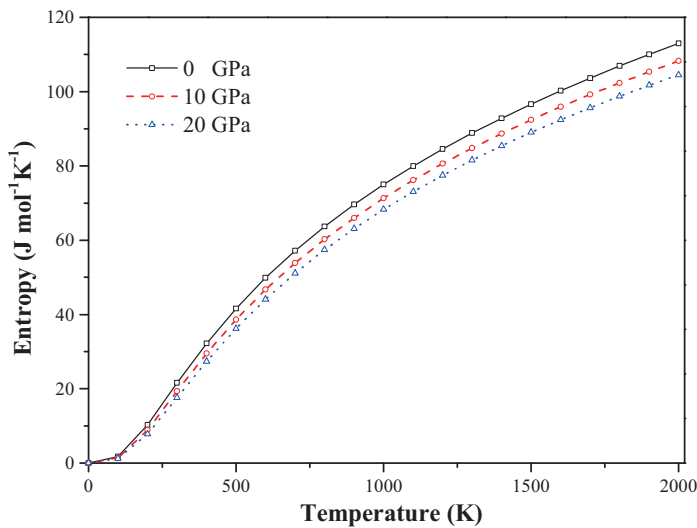

Figure 6. (Color online) Variation of entropy with temperature at a given pressure for TiN.

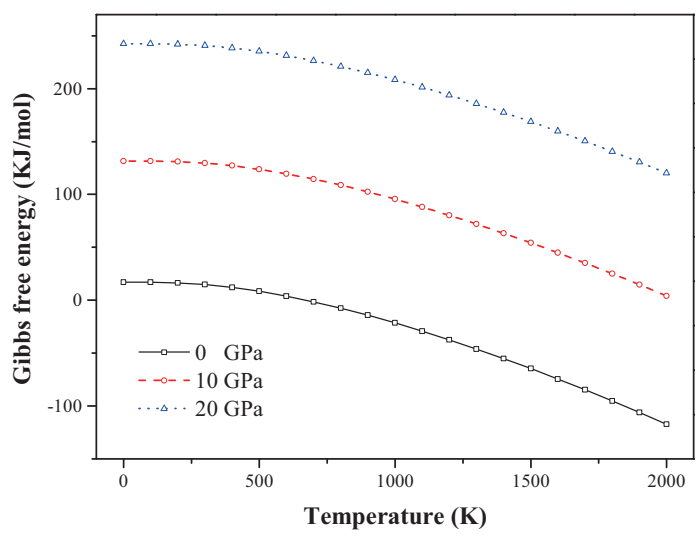

Figure 8. (Color online) The Gibbs energy versus temperature for $\mathrm{TiN}$ at a given pressure.

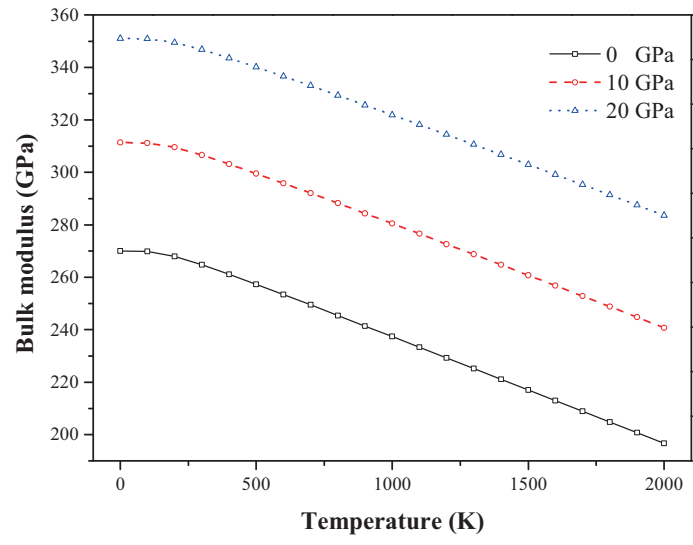

Figure 7. (Color online) The bulk modulus versus temperature for TiN.

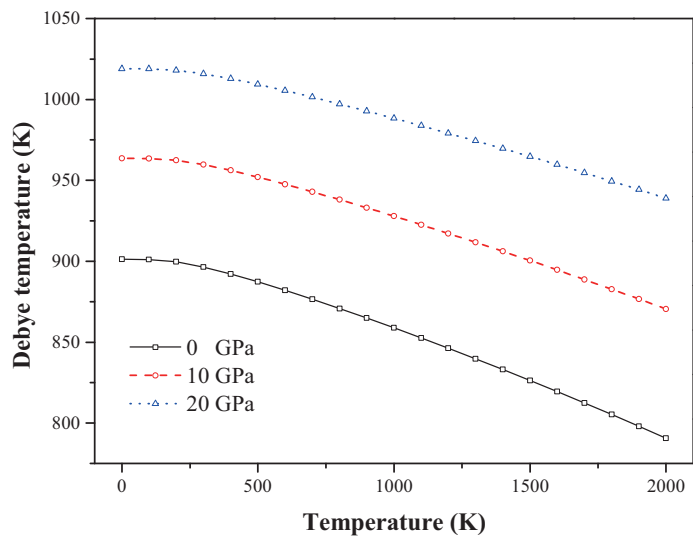

Figure 9. (Color online) The Debye temperature versus temperature at various pressures for TiN.

Debye temperature, $\Theta_{\mathrm{D}}$, is a cut-off on the vibrational modes introduced in the original Debye model. It can facilitate the calculation of the integrals over the phonon density of states within a simple model where only acoustic modes are kept but cut-off at some frequency so that the right number of modes is obtained. It is one of fundamental parameters for solid materials, which is correlated with many physical properties, such as thermal expansion, melting point, and Grüneisen parameter. It is used to distinguish between high and low temperature regions for a solid. At low temperatures, it is proportional to the sound velocity and directly related to the elastic constants through bulk and shear modulus. As the temperature above $\Theta_{\mathrm{D}}$, we expect all modes to have the energy of $\kappa T$ ( $\kappa$ is Boltzmann's constant). At temperatures below it, one expects the high-frequency modes to be frozen, i.e., vibrational excitations arise solely from acoustic vibrations. Figure 9 shows the dependence of the Debye temperature on temperature and pressure. It can be seen that $\Theta_{\mathrm{D}}$ decreases with increasing temperature for TiN, at a given pressure. At a given temperature, it rapidly increases with the applied pressure rise. At zero pressure and zero temperature, the Debye temperature value is equal to $901 \mathrm{~K}$.

\section{Conclusion}

The static dielectric constants of TiN decrease with an increase of pressure from 0 to $29 \mathrm{GPa}$, but it shows an anomalous behavior between 29 and $30 \mathrm{GPa}$, which is interpreted as a first order isostructural phase transition. The absorption spectra exhibit high values ranging from the far infrared region to 
the ultra-violet one. For TiN, $C_{11}$ is the highest in all the elastic constants, which implies that TiN is incompressible along the coordinate axis. The Young's modulus anisotropy ratios are 1.29, 1.44 and 1.58 when the pressures are 0,10 and $20 \mathrm{GPa}$, respectively. Thus, the Young's modulus anisotropy of TiN clearly increases with the pressure rise. Thermodynamic properties of TiN are analyzed in detail by using the quasi-harmonic Debye model under high temperature and pressure. The results show that the effects of pressure and temperature on the bulk modulus, Grüneisen parameter, Gibbs free energy, and Debye temperature are significant. The effect of temperature on the heat capacities and entropy is obvious. At temperature below $1000 \mathrm{~K}$, TiN's heat capacity is larger than that of c-BN. The Grüneisen parameter of $\mathrm{TiN}$ is larger than that of $\mathrm{c}-\mathrm{BN}$.

\section{Acknowledgements}

This work was supported by Natural Science Basic Research plan in Shaanxi Province of China (Grant No. 2016JM1026) and the 111 Project (B17035).

\section{References}

1. Chen C.C., Liang X.T., Tse W.S., Chen I.Y., Duh J.G., Chin. J. Phys., 1994, 32, 205.

2. Kim H.T., Kim M.J., Sohn S.H., J. Phys. Chem. Solids, 2012, 73, 931, doi 10.1016/j.jpcs.2012.02.024

3. Yang R., Zhu C., Wei Q., Du Z., J. Phys. Chem. Solids, 2016, 98, 10, doi 10.1016/j.jpcs.2016.05.012.

4. Guskos N., Bodziony T., Biedunkiewicz A., Aidinis K., Acta Phys. Pol. A, 2006, 108, 311 , doi $10.12693 /$ APhysPolA.108.311.

5. Mashhadi H.A., Kennedy G., Tanaka S., Hokamoto K., Physica B, 2011, 406, 1211, doi $10.1016 /$ j.physb.2010.12.006

6. Wang C., Dai Y., Gao H., Ruan X., Wang J., Sun B., Solid State Commun., 2010, 150, 1370, doi $10.1016 /$ j.ssc.2010.04.034.

7. Zhu B., Li Y.H., Zhu J., Hao Y.J., Xiang G., Yu B.R., Li W., Comput. Mater. Sci., 2014, 86, 200, doi $10.1016 /$ j.commatsci.2014.01.046

8. Li T., Liu T., Wei H., Hussain S., Miao B., Zeng W., Peng X., Pan F., Comput. Mater. Sci., 2015, 105, 83, doi $10.1016 /$ j.commatsci.2015.04.005

9. Yin D., Yang Y., Peng X., Qin Y., Wang Z., Ceram. Int., 2014, 40, 14453, doi $10.1016 /$ j.ceramint.2014.07.016

10. Liu K., Zhou X.L., Chen H.H., Lu L.Y., J. Therm. Anal. Calorim., 2012, 110, 973, doi $10.1007 / \mathrm{s} 10973-011-1927-5$

11. Zhao J.G., Yang L.X., Yu Y., You Sh.J., Yu R.Ch., Li F.Y., Chen L.Ch., Jin Ch.Q., Li X.D., Li Y.Ch., Liu J., Chin. Phys. Lett., 2005, 22, 1199, doi 10.1088/0256-307X/22/5/048.

12. Durandurdu M., Philos. Mag., 2015, 95, 2376, doi:10.1080/14786435.2015.1056855

13. Segall M.D., Lindan P.J.D., Probert M.J., Pickard C.J., Hasnip P.J., Clark S.J., Payne M.C., J. Phys.: Condens. Matter, 2002, 14, 2717, doi 10.1088/0953-8984/14/11/301.

14. Monkhorst H.J., Pack J.D., Phys. Rev. B, 1976, 13, 5188, doi $10.1103 /$ PhysRevB.13.5188

15. Zhang X.D., Shi H.F., Mater. Sci. Technol., 2014, 30, 732, doi:10.1179/1743284713Y.0000000423

16. Jiao Z.Y., Ma S.H., Yang J.F., Solid State Sci., 2011, 13, 331, doi:10.1016/j.solidstatesciences.2010.11.030

17. Shao Z.G., Sun Z.L., Physica E, 2015, 74, 438, doi 10.1016/j.physe.2015.07.011.

18. Misra D., Kundu T.K., Comput. Mater. Sci., 2016, 112, 113, doi 10.1016/j.commatsci.2015.10.021

19. Yu S., Zeng Q., Oganov A.R., Frapper G., Zhang L., Phys. Chem. Chem. Phys., 2015, 17, 11763, doi $10.1039 / \mathrm{c} 5 \mathrm{cp} 00156 \mathrm{k}$

20. Blanco M.A., Francisco E., Luaña V., Comput. Phys. Commun., 2004, 158, 57, doi $10.1016 / j . c o m p h y .2003 .12 .001$

21. Daho S., Ameri M., Al Douri Y., Bensaid D., Varshney D., Ameri I., Mater. Sci. Semicond. Process., 2016, 41, 102, doi 10.1016/j.mssp.2015.08.031.

22. Fan Q., Wei Q., Chai C., Yang Y., Yu X., Liu Y., Zheng J., Zhou P., Zhang D., Acta Phys. Pol. A, 2016, 129, 103, doi 10.12693/APhysPolA.129.103.

23. Wei Q., Zhang M., Guo L., Yan H., Zhu X., Lin Z., Guo P., Chem. Phys., 2013, 415, 36, doi $10.1016 /$ j.chemphys.2013.02.015. 
24. Yan H.Y., Zhang M.G., Huang D.H., Wei Q., Solid State Sci., 2013, 18, 17, doi $10.1016 /$ j.solidstatesciences.2012.12.015

25. Wei Q., Zhang Q., Yan H., Zhang M., J. Mater. Sci., 2017, 52, 2385, doi 10.1007/s10853-016-0564-6.

26. Zhang J.S., Bass J.D., Taniguchi T., Goncharov A.F., Chang Y.-Y., Jacobsen S.D., J. Appl. Phys., 2011, 109, 063521, doi: $10.1063 / 1.3561496$.

27. Fan Q., Wei Q., Chai C., Yan H., Zhang M., Lin Z., Zhang Z., Zhang J., Zhang D., J. Phys. Chem. Solids, 2015, 79, 89, doi:10.1016/j.jpcs.2014.12.008

28. Dincer I., Cengel Y.A., Entropy, 2001, 3, 116, doi 10.3390/e3030116.

\title{
Дослідження з перших принципів оптичних, пружних анізотропних і термодинамічних властивостей TiN при високих температурах і тисках
}

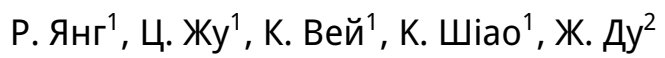 \\ 1 Школа фізики та оптоелектронної інженерії, університет округу Шідіан, Сіань, Шенсі 710071, Китай \\ 2 Національний центр високопродуктивних обчислень, м. Шеньчжень 518055, Китай
}

\begin{abstract}
Детально проаналізовано оптичні, пружні анізотропні та термодинамічні властивості TiN в структурі $\mathrm{NaCl}$ (B1) в температурній області від 0 до $2000 \mathrm{~K} \mathrm{і} \mathrm{в} \mathrm{діапазоні} \mathrm{тисків} \mathrm{від} 0$ до $20 \mathrm{GPa} .3$ обчислених діелектричних постійних знайдено ізоструктурний фазовий перехід першого роду між 29 і $30 \mathrm{GPa}$. Спектр поглинання демонструє високі значення від інфрачервоної області до ультрафіолетової. Значення анізотропії модуля Юнга для TiN $\epsilon$ менше ніж для c-BN при $0 \mathrm{GPa}$, і анізотропія TiN чітко зростає з ростом тиску. Впливи тиску і температури на об'ємний модуль, параметр Грюнайзена, вільну енергію Гіббса і температуру Дебая $\epsilon$ значними. Параметр Грюнайзена для TiN $\epsilon$ набагато більшим ніж для c-BN. При температурах нижчих $1000 \mathrm{~K}$, питома теплоємність TiN $€$ набагато більша ніж с-BN.
\end{abstract}

Ключові слова: TiN, оптичні властивості, пружна анізотропія, термодинамічні властивості, перші принципи 